\title{
Dark origins of matter-antimatter asymmetry
}

\author{
Venus Keus* \\ Department of Physics and Helsinki Institute of Physics, Gustaf Hallstromin katu 2, FIN-00014 \\ University of Helsinki, Finland \\ E-mail: venus.keus@helsinki.fi
}

In a non-minimal Higgs framework, we present a novel mechanism in which the CP violating dark particles only interact with the SM through the gauge bosons, primarily the $Z$ boson. Such $Z$-portal dark CP violation is realised in the regions of the parameter space where Higgs-mediated (co)annihilation processes are sub-dominant and have negligible contributions to the DM relic density. We show that in these regions of the parameter space, the $Z$ portal CP violating DM can still thermalise and satisfy all experimental and observational data. We discuss the implications of such phenomena for electroweak baryogenesis.

Corfu Summer Institute 2019 "School and Workshops on Elementary Particle Physics and Gravity" (CORFU2019)

31 August - 25 September 2019

Corfu, Greece

${ }^{*}$ Speaker. 


\section{Introduction}

The great success of the Standard Model (SM) of particle physics was achieved with the discovery of the long-awaited Higgs boson, its last missing particle, in 2012 at the Large Hadron Collider (LHC) [1, 2], as this particle was predicted by the electroweak symmetry breaking (EWSB) mechanism in 1964 [3, 4]. The SM has been extensively tested and is in great agreement with experiment. So far, the LHC has not detected any significant deviation from the SM, and the observed scalar is very SM-like [5, 6].

Nonetheless, it is widely accepted that the SM is not the ultimate theory of nature due to its shortcomings in explaining several phenomena; many astrophysical observations hint towards the existence of a stable (on cosmological time scales) Dark Matter (DM) particle which is cold (nonrelativistic at the onset of galaxy formation), non-baryonic, neutral and weakly interacting [7]. The particle content of SM does not provide such a candidate. Moreover, the SM lacks a successful baryogenesis mechanism explaining the origin of the observed matter-antimatter asymmetry in the universe. One of the most promising baryogenesis scenarios is electroweak baryogenesis (EWBG) [8], which produces the baryon excess during the electroweak phase transition (EWPT). Although the SM contains all required ingredients for EWBG, it is unable to explain the observed baryon excess due to its insufficient amount of CP violation [9, 10, 11] and the lack of a first-order phase transition [12]. Another shortfall of the SM is that it offers no explanation from underlying physics which could give rise to the fermion masses and mixing patterns; in the SM the fermion masses and mixings are given by Yukawa couplings which are simply parameters to be measured. The indisputable experimental evidence for neutrino masses and oscillations also necessitates going beyond the SM (BSM) framework.

A common characteristic of BSM theories, is an extended scalar sector, suggesting that the observed scalar at the LHC is just one member of an extended scalar sector. The scalar sector in the SM is the experimentally-least-constrained sector, and could provide new sources of $\mathrm{CP}$ violation and a strong first order EWPT if extended. Moreover, non-minimal Higgs sectors with discrete symmetries could naturally accommodate Weakly Interacting Massive Particles (WIMPs) $[13,14,15]$ as viable DM candidates whose stability is ensured by the conservation of the discrete symmetry after EWSB. The EWSB patterns determine the number of active (developing a vacuum expectation value (VEV)) and inert (without a VEV) multiplets in the model.

Simple one-singlet and one-doublet extensions of the SM, i.e. the Higgs portal model [16], the 2-Higgs doublet model (2HDM) (see e.g. [17, 18] and references therein) and the Inert Doublet Model (IDM) [19], have been studied to an advanced level, even though these models by construction can only partly provide a solution to some of the SM drawbacks. In these models, either the scalar potential is inevitably $\mathrm{CP}$-conserving ${ }^{1}$ due to an exact $Z_{2}$ symmetry to stabilise the DM candidate, or CP violation is introduced at the expense of breaking the $Z_{2}$ symmetry and loosing the DM candidate as a result. Nevertheless, such new sources of CP-violation modify the SM-Higgs couplings and contribute to the Electric Dipole Moments (EDMs) of the neutron, electron, and certain atomic nuclei [21] and are, therefore, highly constrained by experiment [22, 23, 24, 25].

\footnotetext{
${ }^{1}$ The purely singlet scalar extension of the SM is CP-conserving regardless of an apparent phase in the potential or the vacuum [20].
} 
One needs to go beyond the simple doublet or singlet scalar extensions of the SM to incorporate both CP-violation and DM into the model. To avoid the pitfalls of the IDM and the Higgs portal model, one needs to extend the inert sector which contains the DM candidate whose stability is ensured by the symmetry of the potential, or remnant thereof after EWSB. If one were to extend the active sector to accommodate CP-violation, one runs into the same limitations as in the 2HDM due to the modification of the SM-Higgs couplings and contributions to the EDMs [26, 27]. On the other hand, if CP violation is introduced in the extended inert sector, there will be no contributions to the EDMs and no limit on the amount of CP violation in the model, since the inert sector is protected from directly coupling to the SM particles.

The phenomena of dark CP-violation and CP violating DM were introduced for the first time in $[28,29,30,31,32]$, and was shown to provide a handle on the otherwise fixed gauge couplings. As a result, a large region of the parameter space opens up to accommodate a DM candidate in agreement with cosmological and collider experiments. Note that a 3-Higgs doublet model (3HDM) with an extended inert sector is superior to a $2 \mathrm{HDM}$ plus a singlet scalar with an extended inert sector [33]; the amount of CP-violation is inevitably reduced due to the presence of the singlet and there exist fewer co-annihilation channels for the DM candidate.

To build a stronger case for $3 \mathrm{HDMs}$, let us point out that not only they contain viable DM candidates, open up a whole new possibility to the CP quantum number of the DM candidate, provide new sources of CP-violation and strongly first order EWPT [34], but also they contain Inflaton candidates driving the inflation process in the beginning of the universe $[35,36]$ and provide a solution to the fermion mass hierarchy problem[37, 38, 39, 40, 41], all in one framework owing to different symmetries that can be imposed on the scalar potential [32]. Here, we expand on the dark $\mathrm{CP}$ violation mechanism, and require the dark/inert particles to interact with the SM only through the gauge bosons, primarily the $Z$ boson. The Higgs-DM couplings is heavily constrained by direct and indirect detection experiments and the LHC bound on the $\mathrm{BR}(h \rightarrow i n v$.) for low DM masses, $m_{D M}<m_{h} / 2$. We show that the $Z$ portal CP-violating DM which is rid of these constraints, can still thermalise and satisfy all experimental and observational data.

In what follows, we present the details of the scalar potential and the theoretical and experimental limits on its parameters in section 2. We construct and justify our benchmark scenarios in section 3. The effect of dark CP-violation on the production and annihilation of DM is discussed in section 4, and finally, we conclude and present the outlook for our future studies in section 5.

\section{The scalar potential with explicit $\mathrm{CP}$ violation}

A 3HDM scalar potential symmetric under a group $Z_{2}$ can be written as the sum of $V_{0}$ with terms symmetric under any phase rotation, and $V_{Z_{2}}$ with terms ensuring the symmetry of the potential $[42,43], V_{3 H D M}=V_{0}+V_{Z_{2}}$, where

$$
\begin{aligned}
V_{0}= & -\mu_{1}^{2}\left(\phi_{1}^{\dagger} \phi_{1}\right)-\mu_{2}^{2}\left(\phi_{2}^{\dagger} \phi_{2}\right)-\mu_{3}^{2}\left(\phi_{3}^{\dagger} \phi_{3}\right)+\lambda_{11}\left(\phi_{1}^{\dagger} \phi_{1}\right)^{2}+\lambda_{22}\left(\phi_{2}^{\dagger} \phi_{2}\right)^{2}+\lambda_{33}\left(\phi_{3}^{\dagger} \phi_{3}\right)^{2} \\
& +\lambda_{12}\left(\phi_{1}^{\dagger} \phi_{1}\right)\left(\phi_{2}^{\dagger} \phi_{2}\right)+\lambda_{23}\left(\phi_{2}^{\dagger} \phi_{2}\right)\left(\phi_{3}^{\dagger} \phi_{3}\right)+\lambda_{31}\left(\phi_{3}^{\dagger} \phi_{3}\right)\left(\phi_{1}^{\dagger} \phi_{1}\right)+\lambda_{12}^{\prime}\left(\phi_{1}^{\dagger} \phi_{2}\right)\left(\phi_{2}^{\dagger} \phi_{1}\right) \\
& +\lambda_{23}^{\prime}\left(\phi_{2}^{\dagger} \phi_{3}\right)\left(\phi_{3}^{\dagger} \phi_{2}\right)+\lambda_{31}^{\prime}\left(\phi_{3}^{\dagger} \phi_{1}\right)\left(\phi_{1}^{\dagger} \phi_{3}\right), \\
V_{Z_{2}}= & -\mu_{12}^{2}\left(\phi_{1}^{\dagger} \phi_{2}\right)+\lambda_{1}\left(\phi_{1}^{\dagger} \phi_{2}\right)^{2}+\lambda_{2}\left(\phi_{2}^{\dagger} \phi_{3}\right)^{2}+\lambda_{3}\left(\phi_{3}^{\dagger} \phi_{1}\right)^{2}+\text { h.c. },
\end{aligned}
$$


where the three Higgs doublets, $\phi_{1}, \phi_{2}, \phi_{3}$, transform under the $Z_{2}$ group as:

$$
\phi_{1} \rightarrow-\phi_{1}, \quad \phi_{2} \rightarrow-\phi_{2}, \quad \phi_{3} \rightarrow+\phi_{3} .
$$

The $Z_{2}$ symmetry is respected by the vacuum,

$$
\left\langle\phi_{1}\right\rangle=0, \quad\left\langle\phi_{2}\right\rangle=0, \quad\left\langle\phi_{3}\right\rangle=\frac{v}{\sqrt{2}},
$$

rendering $\phi_{1}$ and $\phi_{2}$ as the $Z_{2}$-odd inert doublets and $\phi_{3}$ as the $Z_{2}$-even active doublet. The composition of the doublets is as follows,

$$
\phi_{1}=\left(\begin{array}{c}
H_{1}^{+} \\
\frac{H_{1}+i A_{1}}{\sqrt{2}}
\end{array}\right), \quad \phi_{2}=\left(\begin{array}{c}
H_{2}^{+} \\
\frac{H_{2}+i A_{2}}{\sqrt{2}}
\end{array}\right), \quad \phi_{3}=\left(\begin{array}{c}
G^{+} \\
\frac{v+h+i G^{0}}{\sqrt{2}}
\end{array}\right),
$$

where $\phi_{3}$ plays the role of the SM Higgs doublet, with $h$ being the SM Higgs boson and $G^{ \pm}, G^{0}$ the would-be Goldstone bosons.

The parameters of the phase invariant part of the potential, $V_{0}$, are by construction real, while the parameters of $V_{Z_{2}}$, namely $\mu_{12}^{2}, \lambda_{1}, \lambda_{2}, \lambda_{3}$, could be complex and act as a source of explicit CP violation ${ }^{2}$. Thus, the $\mathrm{CP}$-violation is introduced in the inert/dark sector which is forbidden, by the conservation of the $Z_{2}$ symmetry, from mixing with the active sector, and is therefore not limited by EDMs. The lightest particle amongst the CP-mixed neutral fields from the inert doublets is a stable particle and a viable DM candidate. The phenomenologically relevant parameters are $\mu_{3}^{2}, \lambda_{33}$ which are fixed by the Higgs mass, and $\mu_{1}^{2}, \mu_{2}^{2}, \mu_{12}^{2}, \lambda_{31}, \lambda_{23}, \lambda_{31}^{\prime}, \lambda_{23}^{\prime}, \lambda_{2}, \lambda_{3}$ which appear in inert scalars masses and couplings and are, in principle, independent. However, here we limit our study to the dark democracy limit [44, 45, 28, 31], where

$$
\mu_{1}^{2}=\mu_{2}^{2}, \quad \lambda_{3}=\lambda_{2}, \quad \lambda_{31}=\lambda_{23}, \quad \lambda_{31}^{\prime}=\lambda_{23}^{\prime} .
$$

Note that after imposing the dark democracy limit, the model is still explicitly CP-violating since $\left(\lambda_{22}-\lambda_{11}\right)\left[\lambda_{1}\left(\mu_{12}^{2 *}\right)^{2}-\lambda_{1}^{*}\left(\mu_{12}^{2}\right)^{2}\right] \neq 0[46,47]$. Further, one could rotate away the apparent phase of $\mu_{12}^{2}$ by a redefinition of doublets [31]. This leaves $\theta_{2}$, the phase of the $\lambda_{2}$ parameter,

$$
\lambda_{2}=\operatorname{Re} \lambda_{2}+i \operatorname{Im} \lambda_{2}=\left|\lambda_{2}\right| e^{i \theta_{2}},
$$

as the only relevant $\mathrm{CP}$-violating factor which is referred to as $\theta_{\mathrm{CPV}}$ throughout the paper.

\subsection{The mass spectrum}

The minimum of the potential lies at the $\left(0,0, \frac{v}{\sqrt{2}}\right)$ point when $v^{2}=\mu_{3}^{2} / \lambda_{33}$. The fields in the only active doublet, $\phi_{3}$, are mass eigenstates with $G^{0}, G^{ \pm}$as the massless Goldstone bosons, and $h$ as the SM-like Higgs with $m_{h}^{2}=2 \mu_{3}^{2}=2 \lambda_{33} v^{2}=(125 \mathrm{GeV})^{2}$.

The inert doublets mix, resulting in two physical charged states, $S_{1,2}^{ \pm}$, which are a combination of the charged components of the inert doublets, $H_{1,2}^{ \pm}$,

$$
S_{1}^{ \pm}=\frac{H_{1}^{ \pm}+H_{2}^{ \pm}}{\sqrt{2}}, \quad S_{2}^{ \pm}=\frac{H_{1}^{ \pm}-H_{2}^{ \pm}}{\sqrt{2}},
$$

\footnotetext{
${ }^{2}$ Note that $\lambda_{1}$ (and other dark sector parameters $\lambda_{11}, \lambda_{22}, \lambda_{12}, \lambda_{12}^{\prime}$ ) appear only in the inert scalars self-interactions and have no relevance for our DM and collider phenomenology studies. Therefore, for simplicity, their values are set to 0.1 which respects the bounds from perturbative unitarity and positivity of the potential.
} 
with mass-squared values

$$
m_{S_{1}^{ \pm}}^{2}=-\mu_{2}^{2}-\mu_{12}^{2}+\frac{1}{2} \lambda_{23} v^{2}, \quad m_{S_{2}^{ \pm}}^{2}=-\mu_{2}^{2}+\mu_{12}^{2}+\frac{1}{2} \lambda_{23} v^{2},
$$

where require $\mu_{12}^{2}>0$ and fix the hierarchy of the inert charged states, $m_{S_{1}^{ \pm}}<m_{S_{2}^{ \pm}}$. The four inert neutral scalars, $S_{1}, S_{2}, S_{3}, S_{4}$, are a combination of the CP-even and CP-odd components of the inert doublets, $H_{1}, H_{2}, A_{1}, A_{2}$,

$$
\begin{array}{ll}
S_{1}=\frac{\alpha H_{1}-A_{1}+\alpha H_{2}+A_{2}}{\sqrt{2} \sqrt{\alpha^{2}+1}}, & S_{2}=\frac{H_{1}+\alpha A_{1}+H_{2}-\alpha A_{2}}{\sqrt{2} \sqrt{\alpha^{2}+1}}, \\
S_{3}=\frac{\beta H_{1}+A_{1}-\beta H_{2}+A_{2}}{\sqrt{2} \sqrt{\beta^{2}+1}}, & S_{4}=\frac{-H_{1}+\beta A_{1}+H_{2}+\beta A_{2}}{\sqrt{2} \sqrt{\beta^{2}+1}},
\end{array}
$$

where $\alpha$ and $\beta$ are defined as

$$
\alpha=\frac{-\mu_{12}^{2}+v^{2}\left|\lambda_{2}\right| \cos \theta_{C P V}-\Lambda^{-}}{v^{2}\left|\lambda_{2}\right| \sin \theta_{C P V}}, \quad \beta=\frac{-\mu_{12}^{2}-v^{2}\left|\lambda_{2}\right| \cos \theta_{C P V}+\Lambda^{+}}{v^{2}\left|\lambda_{2}\right| \sin \theta_{C P V}},
$$

and $\Lambda^{\mp}$ as

$$
\Lambda^{\mp}=\sqrt{\left(\mu_{12}^{2}\right)^{2}+v^{4}\left|\lambda_{2}\right|^{2} \mp 2 v^{2} \mu_{12}^{2}\left|\lambda_{2}\right| \cos \theta_{C P V}} .
$$

The masses of the neutral CP-mixed inert scalars, $S_{1}, S_{2}, S_{3}, S_{4}$, are calculated to be

$$
m_{S_{1,2}}^{2}=-\mu_{2}^{2}+\frac{v^{2}}{2}\left(\lambda_{23}^{\prime}+\lambda_{23}\right) \mp \Lambda^{-}, \quad m_{S_{3,4}}^{2}=-\mu_{2}^{2}+\frac{v^{2}}{2}\left(\lambda_{23}^{\prime}+\lambda_{23}\right) \mp \Lambda^{+} .
$$

As the DM candidate, we require $S_{1}$ to be lightest inert particle which leads to

$$
m_{S_{1}}<m_{S_{2}}, m_{S_{3}}, m_{S_{4}} \quad \Rightarrow \quad \frac{\pi}{2}<\theta_{C P V}<\frac{3 \pi}{2},
$$

when $\lambda_{2}<0$ in agreement with $[44,45]^{3}$. At $\theta_{C P V}=\frac{\pi}{2}, \frac{3 \pi}{2}$ where $\Lambda^{+}=\Lambda^{-}$, a mass degeneracy between neutral inert particles occurs, $m_{S_{1}}=m_{S_{3}}$ and $m_{S_{2}}=m_{S_{4}}$. At $\theta_{C P V}=0, \pi$, the model is reduced to the CP-conserving limit which renders $S_{1,3}$ to CP-even and $S_{2,4}$ to CP-odd particles,

$$
\theta_{C P V}=0, \pi \quad \Rightarrow \quad \text { CP-conserving limit : }\left\{\begin{array}{l}
S_{1,3}=\frac{H_{1} \pm H_{2}}{\sqrt{2}}, \\
S_{2,4}=\frac{A_{1} \pm A_{2}}{\sqrt{2}} .
\end{array}\right.
$$

We take all other parameters of the potential to be positive. The independent parameters, $\mu_{12}^{2}, \lambda_{23}$, $\lambda_{23}^{\prime}, \mu_{2}^{2}, \lambda_{2}$, are written in terms of scalar masses and couplings, $m_{S_{1}}, m_{S_{2}}, m_{S_{1}^{ \pm}}, m_{S_{2}^{ \pm}}, g_{h D M}$, which are taken as the input parameters of the model, where $g_{h D M} \equiv g_{S_{1} S_{1} h}$ is the Higgs-DM coupling, with the relevant terms in the Lagrangian appearing as:

$$
\mathscr{L} \supset g_{Z S_{i} S_{j}} Z_{\mu}\left(S_{i} \partial^{\mu} S_{j}-S_{j} \partial^{\mu} S_{i}\right)+\frac{v}{2} g_{S_{i} S_{i} h} h S_{i}^{2}+v g_{S_{i} S_{j} h} h S_{i} S_{j}+v g_{S_{i}^{ \pm} S_{j}^{\mp}} h S_{i}^{ \pm} S_{j}^{\mp} .
$$

${ }^{3}$ If $\theta_{C P V}$ is taken to be in the $2^{\text {nd }}$ quadrant, for $\lambda_{2}>0$ the above augments hold provided the neutral inert particles are relabelled as $S_{1} \leftrightarrow S_{3}$ and $S_{2} \leftrightarrow S_{4}$, similarly, if $\theta_{C P V}$ is taken to be in the $1^{\text {st }}$ or $4^{\text {th }}$ quadrants for $\lambda_{2}<0$. 


\subsection{Constraints on the parameter space}

The parameter space of the model is constrained by:

1. theoretical bounds: boundedness-from-below of the potential, positive-definiteness of the Hessian, perturbative unitarity and electroweak oblique $S, T, U$ parameters

2. experimental bounds: total decay width of $W^{ \pm}, Z$ bosons, non-observation of charged scalars, Higgs total decay width and Higgs signal strengths, Higgs invisible branching ratio for light inert scalars,

3. observational bounds: relic density measurements and direct and indirect detection of DM, which are satisfied in all our benchmark scenarios as detailed in [32].

\section{The abundance of DM and benchmark selection}

The solution of the Boltzmann equation after freeze out, determines the relic abundance of the DM candidate, $S_{1}$ :

$$
\frac{d n_{S_{1}}}{d t}=-3 H n_{S_{1}}-\left\langle\sigma_{e f f} v\right\rangle\left[\left(n_{S_{1}}\right)^{2}-\left(n_{S_{1}}^{e q}\right)^{2}\right],
$$

where $n_{S_{1}}\left(n_{S_{1}}^{e q}\right)$ is the number density of the $S_{1}$ particle (at equilibrium), and $H$ is the Hubble parameter. The thermally averaged cross section, $\left\langle\sigma_{e f f} v\right\rangle$, receives contribution from all relevant (co)annihilation processes of any $S_{i} S_{j}$ pair into SM particles, so that

$$
\left\langle\sigma_{e f f} v\right\rangle=\sum_{i, j}\left\langle\sigma_{i j} v_{i j}\right\rangle \frac{n_{S_{i}}^{e q}}{n_{S_{1}}^{e q}} \frac{n_{S_{j}}^{e q}}{n_{S_{1}}^{e q}}, \quad \text { where } \quad \frac{n_{S_{i}}^{e q}}{n_{S_{1}}^{e q}} \sim \exp \left(-\frac{m_{S_{i}}-m_{S_{1}}}{T}\right),
$$

with the main contribution from processes where $m_{S_{i}}-m_{S_{1}}$ is comparable to the thermal bath temperature $T$.

A common feature of non-minimal Higgs DM models is that in a large region of the parameter space the dominant process for DM annihilation is $S_{1} S_{1} \rightarrow h_{\mathrm{SM}} \rightarrow f \bar{f}$ whose efficiency depends both on the DM mass and the Higgs-DM coupling. In the low mass region, $m_{\mathrm{DM}}<m_{h} / 2$, generally one requires a large Higgs-DM coupling for an effective DM annihilation leading to a relic density in agreement with observations. However, such large Higgs-DM couplings result in large direct and indirect detection cross sections and significant deviations from SM-Higgs coupling measurements, and hence are ruled out by experimental and observational data. On the other hand, a small HiggsDM coupling, fails to annihilate DM effectively and results in the over-closure of the universe. Here, the co-annihilation processes play an important role as they can contribute to changes in the relic density of DM.

In models with extended dark sectors, in addition to the standard Higgs mediated DM annihilation channels, there exist such co-annihilation channels of DM with heavier states, provided they are close in mass [28, 30, 31, 44, 45]. The relevance of these processes depends not only on the DM mass and the mass splittings, but also on the strength of the standard DM annihilation process. It is worth emphasising that in the IDM, which is by construction CP conserving, the only possible co-annihilation process is through the $Z$-mediated $H A \rightarrow Z \rightarrow f \bar{f}$ channel whose sub-dominant 
effect fails to rescue the model in the low mass region where it is in severe tension with direct and indirect detection bounds and $\mathrm{BR}(h \rightarrow$ inv. $)$ limits.

Extending the inert sector, as shown in $[30,44,45]$ already in the CP-conserving limit, allows for several co-annihilation channels, both Higgs-mediated $H_{1} H_{2} \rightarrow h \rightarrow f \bar{f}$ and $Z$-mediated $H_{1} A_{1,2} \rightarrow Z \rightarrow f \bar{f}$. The collective contribution of these channels to DM co-annihilation is not sufficient to completely rid the model of experimental and observational constraints, since one still needs a non-zero Higgs-DM coupling to satisfy relic density bounds. Introducing CP-violation in the extended dark sector [28, 31, 48], triumphantly opens up many co-annihilation channels through the Higgs and $Z$ bosons, $S_{i} S_{j} \rightarrow h / Z \rightarrow f \bar{f}$, which can significantly affect the DM phenomenology. In fact, the $Z$-mediated co-annihilations can be strong enough to relieve the model of the need for any Higgs-mediated (co)annihilation processes [32].

Focusing on the regions of the parameter space where the Higgs-DM coupling is negligibly small, $g_{h D M} \sim 0$, we highlight the effect of $Z$ portal CP-violation on the abundance of DM. Here, the main DM annihilation process, $S_{1} S_{1} \rightarrow h \rightarrow f \bar{f}$ and, as a result, the Higgs-mediated co-annihilation processes are sub-dominant and have negligible contributions to the DM relic density. Therefore, the only effective communication between the dark sector and the visible sector is through the gauge bosons $W^{ \pm}$and $Z$.

Let us emphasise that the phenomenon of dark $\mathrm{CP}$ violation, is not realisable in purely scalar singlet extensions of the SM. An extended dark sector with a doublet plus a singlet could, in principle, accommodate dark CP-violation, however, the presence of the singlet dilutes the $\mathrm{CP}$ violating effects, since a singlet has no direct coupling to SM gauge bosons. As a result, the model fails to provide a DM candidate accounting for $100 \%$ of the observed relic density in the low mass region [33] We would like to point out that this is the reason Ref. [33] fails to find a DM candidate accounting for $100 \%$ of the observed relic density in the low mass region. Furthermore, the collider signatures of dark CP violation through the $Z Z Z$ vertex and cross section asymmetries [31, 49, 50], in their model is considerably smaller.

With the negligible Higgs mediated processes, the total DM annihilation cross section is determined by the following gauge boson mediated processes, $V=Z, W^{ \pm}$:

- DM annihilation processes:

$$
S_{1} S_{1} \rightarrow V V, \quad S_{1} S_{1} \rightarrow V V^{*} \rightarrow V f f^{\prime}, \quad S_{1} S_{1} \rightarrow V^{*} V^{*} \rightarrow f f^{\prime} f f^{\prime},
$$

where the processes with off-shell gauge bosons dominate over the ones with on-shell gauge bosons, in the $m_{D M}<m_{W}$ region.

\section{- DM co-annihilation processes:}

$$
S_{1} S_{2,3,4} \rightarrow Z^{*} \rightarrow f \bar{f}, \quad S_{1} S_{1,2}^{ \pm} \rightarrow W^{ \pm *} \rightarrow f f^{\prime},
$$

where the co-annihilating dark scalars are up to $20 \%$ heavier than the DM candidate.

- (co)annihilation of other dark states:

$$
S_{i} S_{i} \rightarrow V V, \quad S_{i} S_{i} \rightarrow V V^{*} \rightarrow V f f^{\prime}, \quad S_{i} S_{i} \rightarrow V^{*} V^{*} \rightarrow f f^{\prime} f f^{\prime}, \quad S_{i} S_{j} \rightarrow V^{*} \rightarrow f f^{\prime},
$$

where $S_{i} \neq S_{j}$ are any of the dark scalars $S_{2,3,4}, S_{1,2}^{ \pm}$which are all close in mass. 
Taking all the above processes into account, we define the following characteristic benchmark scenarios with distinct DM phenomenology. We introduce the notations

$$
\delta_{12}=m_{S_{2}}-m_{S_{1}}, \quad \delta_{c}=m_{S_{2}^{ \pm}}-m_{S_{1}^{ \pm}}, \quad \delta_{1 c}=m_{S_{1}^{ \pm}}-m_{S_{1}},
$$

representing the mass splittings between the DM candidate and other inert scalars.

\section{Benchmarks of type 1:}

In the low mass region, $45 \mathrm{GeV}<m_{S_{1}} \leq 80 \mathrm{GeV}$, we devise benchmark scenarios of type 1:

$$
\begin{aligned}
\mathbf{B}_{1} \mathbf{D}_{4} \mathbf{C}_{1}: & \delta_{12}=4 \mathrm{GeV}, \quad \delta_{c}=1 \mathrm{GeV}, \quad \delta_{1 c}=50 \mathrm{GeV}, \\
\mathbf{B}_{1} \mathbf{D}_{8} \mathbf{C}_{1}: & \delta_{12}=8 \mathrm{GeV}, \quad \delta_{c}=1 \mathrm{GeV}, \quad \delta_{1 c}=50 \mathrm{GeV}, \\
\text { where } & m_{S_{1}} \sim m_{S_{3}} \sim m_{S_{2}} \sim m_{S_{4}} \ll m_{S_{1}^{ \pm}} \sim m_{S_{2}^{ \pm}},
\end{aligned}
$$

with all neutral inert particles close in mass, and much lighter than the inert charged particles. We define another two benchmark scenarios of type 1 with a larger $\delta_{12}$, where the neutral inert particles split into two groups, with $S_{1}$ and $S_{3}$ close in mass and lighter than $S_{2}$ and $S_{4}$ which are also close in mass, and with all neutral inert scalars lighter than the charged inert scalars,

$$
\begin{aligned}
\mathbf{B}_{1} \mathbf{D}_{12} \mathbf{C}_{1}: & \delta_{12}=12 \mathrm{GeV}, \quad \delta_{c}=1 \mathrm{GeV}, \quad \delta_{1 c}=50 \mathrm{GeV} \\
\mathbf{B}_{1} \mathbf{D}_{20} \mathbf{C}_{1}: & \delta_{12}=20 \mathrm{GeV}, \quad \delta_{c}=1 \mathrm{GeV}, \quad \delta_{1 c}=50 \mathrm{GeV} \\
\text { where } & m_{S_{1}} \sim m_{S_{3}} \lesssim m_{S_{2}} \sim m_{S_{4}} \ll m_{S_{1}^{ \pm}} \sim m_{S_{2}^{ \pm}} .
\end{aligned}
$$

\section{Benchmarks of type 2:}

In the low mass region, $45 \mathrm{GeV}<m_{S_{1}} \leq 80 \mathrm{GeV}$, we devise two benchmark scenarios of type 2 :

$$
\begin{aligned}
\mathbf{B}_{2} \mathbf{D}_{55} \mathbf{C}_{1}: & \delta_{12}=55 \mathrm{GeV}, \quad \delta_{c}=1 \mathrm{GeV}, \quad \delta_{1 c}=50 \mathrm{GeV}, \\
\mathbf{B}_{2} \mathbf{D}_{55} \mathbf{C}_{15}: & \delta_{12}=55 \mathrm{GeV}, \quad \delta_{c}=15 \mathrm{GeV}, \quad \delta_{1 c}=50 \mathrm{GeV}, \\
\text { where } & m_{S_{1}} \sim m_{S_{3}} \ll m_{S_{2}} \sim m_{S_{4}} \sim m_{S_{1}^{ \pm}} \sim m_{S_{2}^{ \pm}}
\end{aligned}
$$

where only one neutral inert particle, $S_{3}$, is close in mass with the DM candidate, $S_{1}$.

\section{Benchmarks of type 3:}

In the heavy mass region $m_{S_{1}} \geq 80 \mathrm{GeV}$, where the DM candidate can be close in mass with the charged inert particles, we define three benchmark scenarios

$$
\begin{aligned}
\mathbf{B}_{3} \mathbf{D}_{5} \mathbf{C}_{1}: & \delta_{12}=5 \mathrm{GeV}, \quad \delta_{c}=1 \mathrm{GeV}, \quad \delta_{1 c}=1 \mathrm{GeV}, \\
\text { where } & m_{S_{1}} \sim m_{S_{3}} \sim m_{S_{2}} \sim m_{S_{4}} \sim m_{S_{1}^{ \pm}} \sim m_{S_{2}^{ \pm}}, \\
\mathbf{B}_{3} \mathbf{D}_{55} \mathbf{C}_{1}: & \delta_{12}=55 \mathrm{GeV}, \quad \delta_{c}=1 \mathrm{GeV}, \quad \delta_{1 c}=1 \mathrm{GeV}, \\
\text { where } & m_{S_{1}} \sim m_{S_{3}} \sim m_{S_{1}^{ \pm}} \sim m_{S_{2}^{ \pm}} \ll m_{S_{2}} \sim m_{S_{4}}, \\
\mathbf{B}_{3} \mathbf{D}_{55} \mathbf{C}_{22}: & \delta_{12}=55 \mathrm{GeV}, \quad \delta_{c}=22 \mathrm{GeV}, \quad \delta_{1 c}=1 \mathrm{GeV}, \\
\text { where } & m_{S_{1}} \sim m_{S_{3}} \sim m_{S_{1}^{ \pm}} \ll m_{S_{2}^{ \pm}} \sim m_{S_{2}} \sim m_{S_{4}} .
\end{aligned}
$$




\section{The effect of dark CP-violation on the DM abundance}

\subsection{Benchmarks of type 1}

Benchmark scenarios of type 1, represent regions of the parameter space where all neutral inert particles are relatively close in mass and are much lighter than the inert charged particles. Therefore, the main co-annihilation channel in these scenarios is through the $S_{i} S_{j} \rightarrow Z^{*} \rightarrow f \bar{f}$ processes. In $\mathrm{B}_{1} \mathrm{D}_{4} \mathrm{C}_{1}$ and $\mathrm{B}_{1} \mathrm{D}_{8} \mathrm{C}_{1}$ scenarios, with very small $S_{1}-S_{2}$ mass splitting, the co-annihilation of $\mathrm{DM}$ with other neutral scalars is so strong that $\mathrm{DM}$ is under-produced irrespective of size of the $\mathrm{CP}$ violating angle, $\theta_{C P V}$. In $\mathrm{B}_{1} \mathrm{D}_{12} \mathrm{C}_{1}$ and $\mathrm{B}_{1} \mathrm{D}_{20} \mathrm{C}_{1}$ scenarios, with a larger $S_{1}-S_{2}$ mass splitting, these co-annihilation processes are weakened and the $S_{1}$ relic abundance is increased as a result. The efficiency of the co-annihilation processes is also dependant on the strength of the $Z S_{i} S_{j}$ coupling. Figure 1 shows the strength of the relevant and non-negligible $Z S_{i} S_{j}$ couplings in all four type 1 scenarios for an exemplary $m_{S_{1}}$ of $57 \mathrm{GeV}$. As expected the $g_{Z S_{1} S_{3}}$ coupling vanishes at $\theta_{C P V}=\pi$ which is the CP-conserving limit where $S_{1}$ and $S_{3}$ are reduced to two CP-even particles as shown in eq.(2.14).
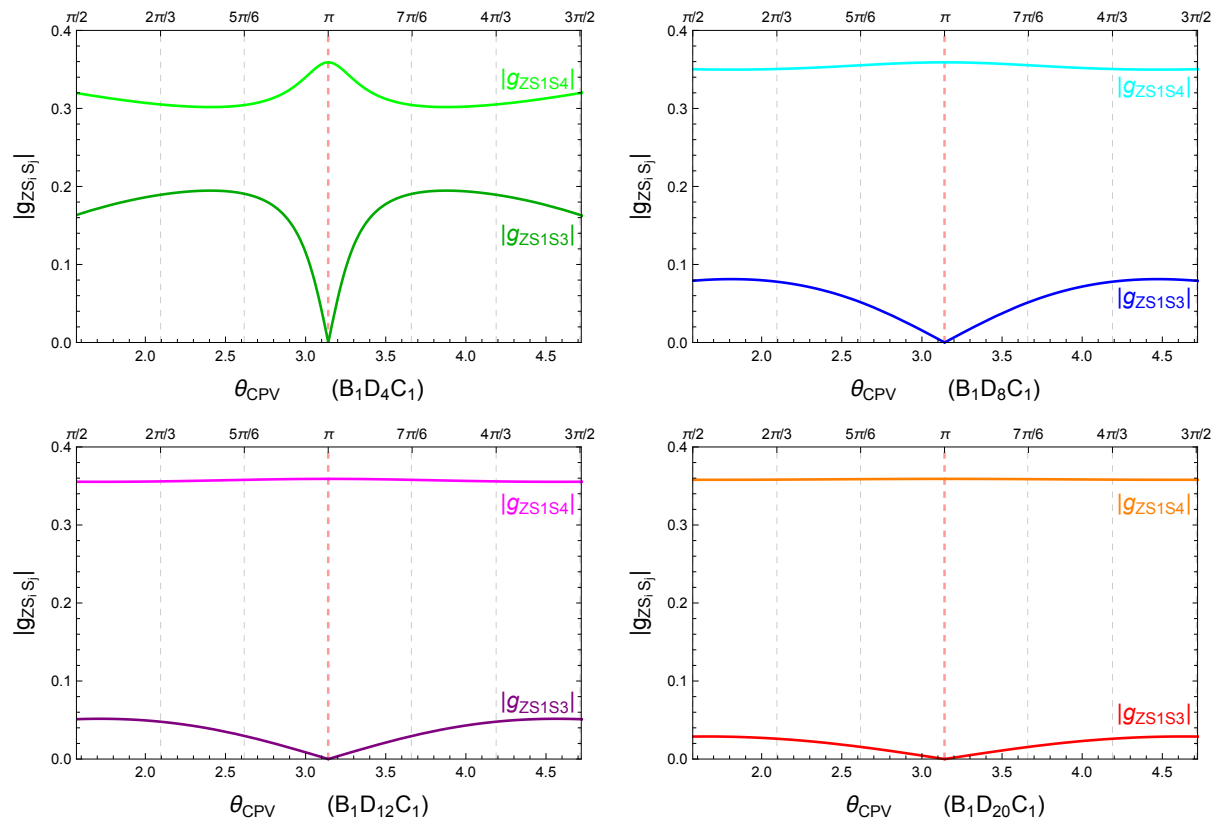

Figure 1: The relevant $Z S_{i} S_{j}$ couplings in type 1 scenarios for an exemplary $m_{S_{1}}=57 \mathrm{GeV}$.

Note that as $\delta_{12}$ increases from $4 \mathrm{GeV}$ in $\mathrm{B}_{1} \mathrm{D}_{4} \mathrm{C}_{1}$ to $20 \mathrm{GeV}$ in $\mathrm{B}_{1} \mathrm{D}_{20} \mathrm{C}_{1}$, the co-annihilation probability of $S_{1}$ with other neutral dark particles is reduced, also the coupling of the main coannihilation channel, $g_{Z S_{1} S_{3}}$ is reduced. As a result, the DM abundance is considerably larger in scenarios with larger $\delta_{12}$, which is well represented in figure 2 .

It is worth noting that in a given benchmark scenario as DM mass increases to values comparable with $m_{W^{ \pm}}$and $m_{Z}$, the $S_{i} S_{i} \rightarrow V V$ annihilation channels come into play and reduce the DM number density. As a result, DM is always under-produced for $m_{S_{1}} \gtrsim 80 \mathrm{GeV}$. Figure 3 shows the regions with correct abundance and under-abundance of $D M$ in $\mathrm{B}_{1} \mathrm{D}_{12} \mathrm{C}_{1}$ and $\mathrm{B}_{1} \mathrm{D}_{20} \mathrm{C}_{1}$ scenarios. In the other type 1 scenarios, $\mathrm{B}_{1} \mathrm{D}_{4} \mathrm{C}_{1}$ and $\mathrm{B}_{1} \mathrm{D}_{8} \mathrm{C}_{1}$, DM is always under-produced. At 

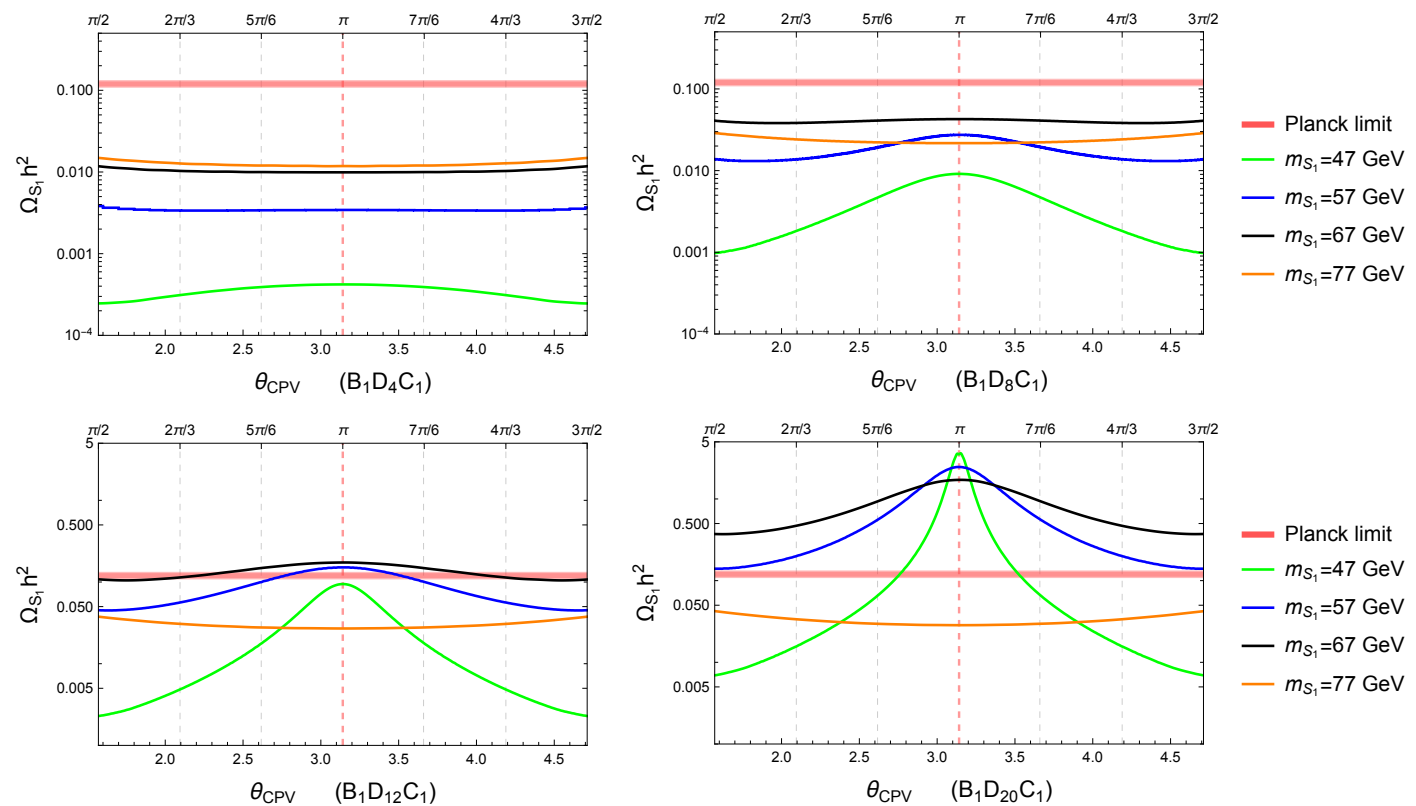

Figure 2: The relic abundance of $S_{1}$ for different DM masses in type 1 benchmark scenarios. The horizontal red band shows the Planck observation limit on the abundance of DM.

$\theta_{C P V}=\pi$, where the model is reduced to the CP conserving limit, the value of $g_{S_{i} S_{j} Z}$ is fixed as a gauge coupling. With no handle on the $S_{i} S_{j} Z$ couplings, DM is over-produced in the intermediate mass region $54 \mathrm{GeV} \lesssim m_{S_{1}} \lesssim 70 \mathrm{GeV}$. Varying the $\mathrm{CP}$ violating phase, $\theta_{C P V}$, can change the strength of the $S_{i} S_{j} Z$ couplings and, as a result, the $S_{1}-S_{3}$ mass splitting. The smallest $S_{1}-S_{3}$ mass splitting occurs close to the $\pi / 2$ and $3 \pi / 2$ boundaries. Therefore, one expects a more effective $S_{1}-S_{3}$ co-annihilation and a smaller relic density as $\theta_{C P V}$ moves away from the CP conserving limit and towards the maximum $\mathrm{CP}$ violation at the $\pi / 2$ and $3 \pi / 2$ boundaries. Figure 3 illustrates this behaviour for $\mathrm{B}_{1} \mathrm{D}_{12} \mathrm{C}_{1}$ and $\mathrm{B}_{1} \mathrm{D}_{20} \mathrm{C}_{1}$ scenarios, where in the latter scenario with a large $\delta_{12}$, the intermediate mass region over-produces DM irrespective of the size of the $S_{1}-S_{3}$ mass splitting.
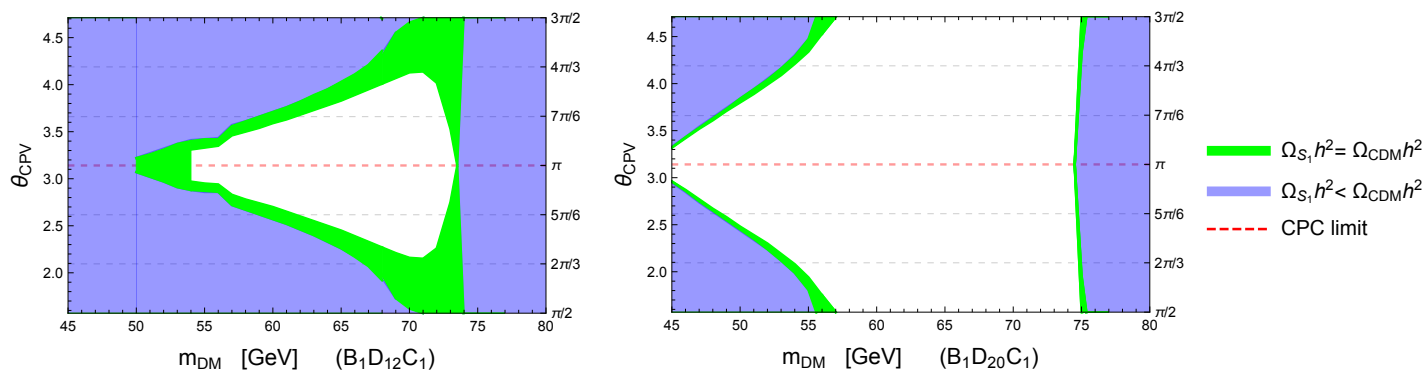

Figure 3: Regions producing $100 \%$ of DM in green, and under-producing DM in blue in the $\theta_{C P V^{-}}$ $m_{D M}$ plane in type 1 scenarios. The horizontal dashed red line represents the $\mathrm{CP}$ conserving limit.

\subsection{Benchmarks of type 2}

In type 2 benchmark scenarios, $\mathrm{B}_{2} \mathrm{D}_{55} \mathrm{C}_{1}$ and $\mathrm{B}_{2} \mathrm{D}_{55} \mathrm{C}_{15}$, only $S_{3}$ is close in mass with $S_{1}$ and 
can co-annihilate with it through the $S_{1} S_{3} \rightarrow Z^{*} \rightarrow f \bar{f}$ process, which dictates the behaviour of the model in the low mass region. As the DM mass approaches the $W^{ \pm}, Z$ masses, the $S_{1} S_{1} \rightarrow V V$ channels effectively annihilate the DM candidate leading to an insufficient relic density for $m_{D M}$ above this range, irrespective of the CP violating angle. Recall that the $Z S_{1} S_{3}$ coupling is sensitive to the changes in the $\mathrm{CP}$ violating angle. Figure 4 shows the absolute value of the $Z S_{1} S_{3}$ coupling for an exemplary DM mass of $50 \mathrm{GeV}$ with respect to $\theta_{C P V}$ in type 2 benchmark scenarios.
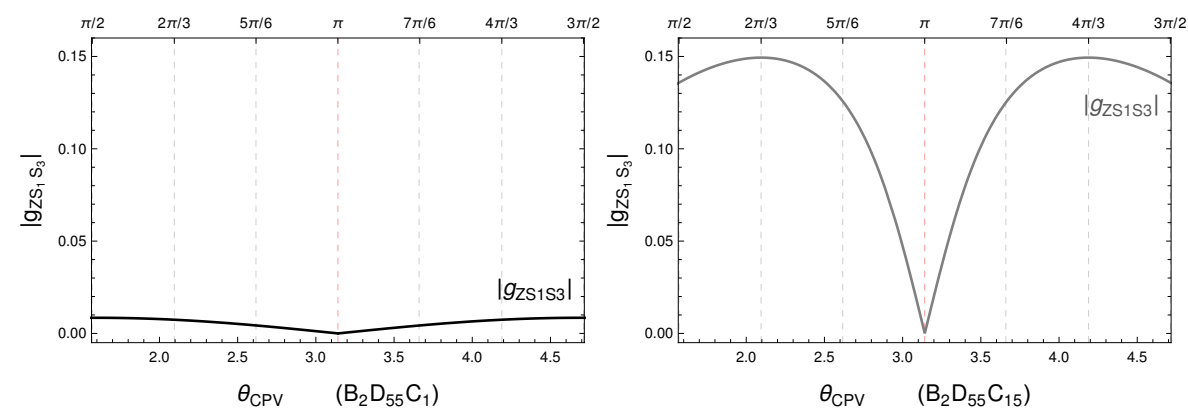

Figure 4: The $g_{Z S_{1} S_{3}}-\theta_{C P V}$ dependence in type 2 benchmark scenarios for a given $m_{S_{1}}=50 \mathrm{GeV}$.

Due to the striking difference in the values of the $g_{Z S_{1} S_{3}}$ coupling in the two benchmark scenarios, one expects a substantial difference in the DM relic density in each case. In the mass range $45 \mathrm{GeV}<m_{D M}<75 \mathrm{GeV}$, the $\mathrm{B}_{2} \mathrm{D}_{55} \mathrm{C}_{1}$ scenario consistently over-produces DM (except for large $\mathrm{CP}$ violating angles around the $Z$ resonance region $m_{D M} \approx m_{Z} / 2$ where $S_{1}$ and $S_{3}$ are very close in mass) since the $Z S_{1} S_{3}$ coupling is so weak that it fails to co-annihilate DM effectively. The $\mathrm{B}_{2} \mathrm{D}_{55} \mathrm{C}_{15}$ scenario, on the other hand, has a large enough $Z S_{1} S_{3}$ coupling at large $\theta_{C P V}$ to satisfy the Planck limit on the DM relic density. Figure 5 illustrates this behaviour where the relic density for various $\mathrm{DM}$ masses is shown. The $\mathrm{B}_{2} \mathrm{D}_{55} \mathrm{C}_{1}$ scenario over-produces DM for masses below 75 $\mathrm{GeV}$, while $\mathrm{B}_{2} \mathrm{D}_{55} \mathrm{C}_{15}$ scenario produces $\mathrm{DM}$ in agreement with the Planck limit for large CP violating angles in this mass range. As mentioned before, both scenarios under-produce DM for larger masses when the $S_{1} S_{1} \rightarrow V V$ annihilation channel is open.
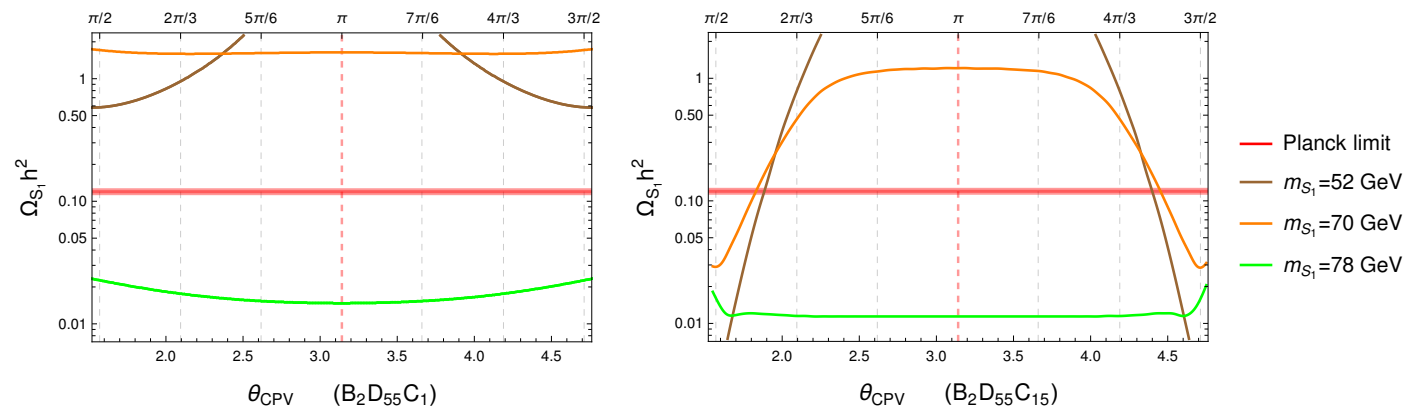

Figure 5: The change in DM relic density for various DM masses with respect to the CP-violating angle. The horizontal red band shows the Planck observation limit on the abundance of DM.

In figure 6 , we show regions where $S_{1}$ contributes to $100 \%$ of the observed DM in green, and regions where it only provides a fraction of the observed relic density in blue, in the $\theta_{C P V}-m_{D M}$ 
plane. The blank regions are ruled out by Planck observations as they lead to an over-production of DM. Note that in the CP conserving limit where $\theta_{C P V}=\pi$ the model fails to comply with the Planck observations throughout the low mass regime.
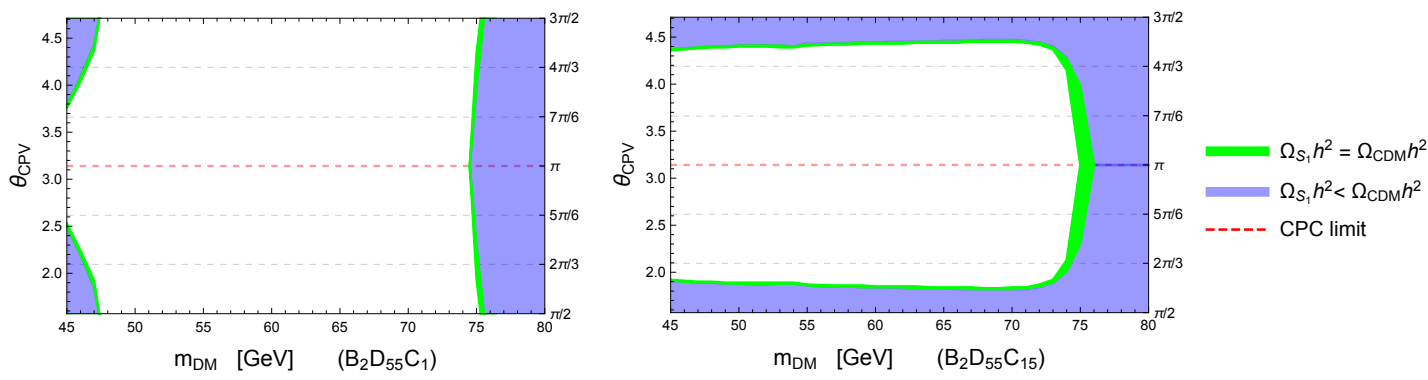

Figure 6: Regions producing $100 \%$ of DM in green, and under-producing DM in blue in the $\theta_{C P V^{-}}$ $m_{D M}$ plane in type 2 scenarios. The horizontal dashed red line represents the $\mathrm{CP}$-conserving limit.

\subsection{Benchmarks of type 3}

The type 3 benchmark scenarios which are defined in the heavy mass region, $m_{D M} \geqslant 80 \mathrm{GeV}$, allow for $S_{1}$ to be close in mass with the charged inert scalars, thereby providing new co-annihilation channels for the DM candidate.
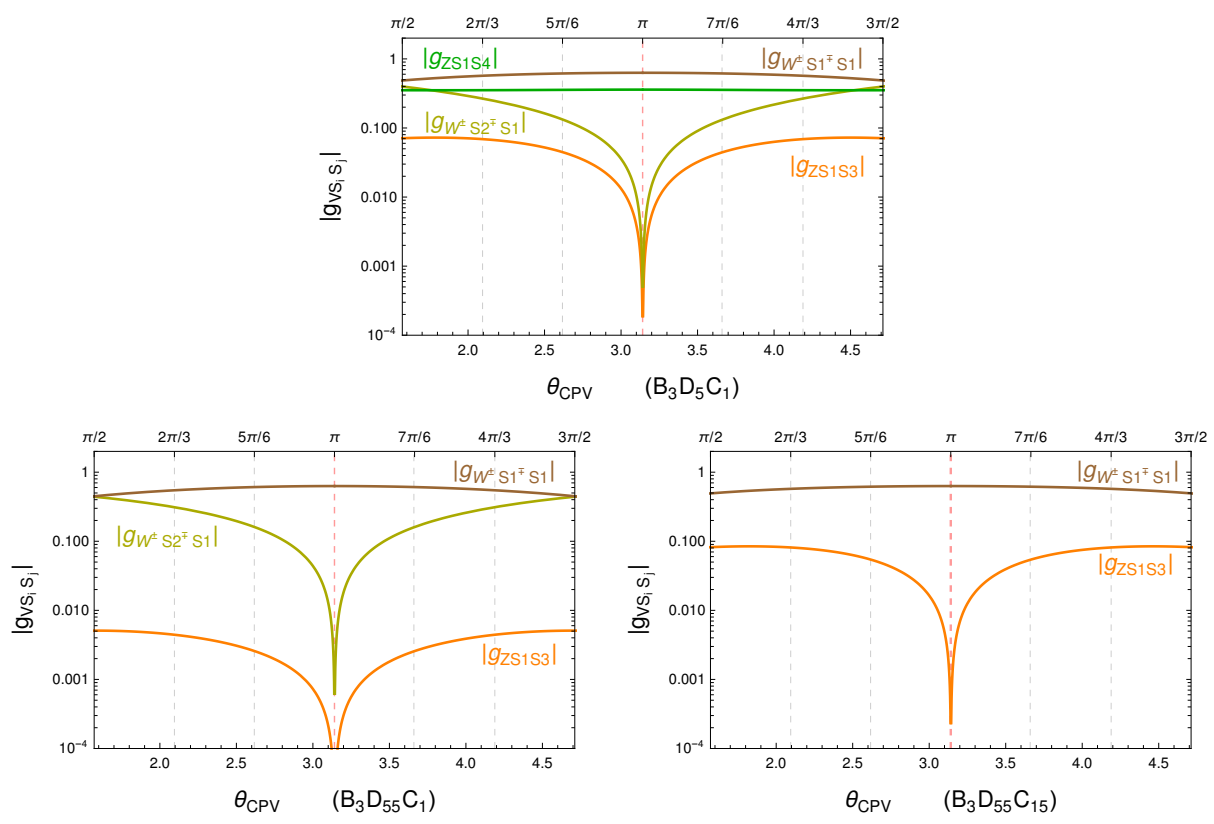

Figure 7: The $g_{V S_{i} S_{j}}-\theta_{C P V}$ dependence in type 3 benchmark scenarios for a given $m_{S_{1}}=90 \mathrm{GeV}$.

When studying the DM phenomenology of the model, it is not only the annihilation and coannihilation of DM, but also the (co)annihilation of other inert particles that should be taken into account. Figure 7 shows the relevant and non-negligible $V S_{1} S_{i}$ couplings for all three type 3 benchmark scenarios where $V=W^{ \pm}, Z$ and $S_{i}$ is a neutral or charged inert particle. Due to the presence 
of so many co-annihilation processes, type 3 scenarios consistently under-produce DM. Moreover, in the heavy mass region the annihilation $S_{1} S_{1} \rightarrow V V$ whose coupling is independent of the CP violating angle, is very effective and leads to the under-production of DM in all three scenarios.

Figure 8 illustrates the relic abundance for various DM masses. As mentioned before, all three scenarios have a similar behaviour, with the $\mathrm{B}_{3} \mathrm{D}_{55} \mathrm{C}_{1}$ scenario providing a slightly larger relic density in comparison to the $\mathrm{B}_{3} \mathrm{D}_{5} \mathrm{C}_{1}$ scenario due to a larger $\delta 12$ mass splitting. Scenario $\mathrm{B}_{3} \mathrm{D}_{55} \mathrm{C}_{15}$ provides only two co-annihilation channels for $S_{1}$, however, they have larger couplings compared to the same processes in the $\mathrm{B}_{3} \mathrm{D}_{55} \mathrm{C}_{1}$ case, which leads to a slightly smaller relic abundance for $S_{1}$ in the former scenario. Let us point out that the reason we do not see the revival of the very heavy mass region $m_{D M}>400 \mathrm{GeV}$ as shown in [45] and [28] is the absence of the Higgs mediated processes $S_{i} S_{j} \rightarrow h \rightarrow V V$, where $S_{i, j}$ is any neutral or charged inert particle. These Higgs-mediated processes have a destructive interference with pure gauge processes $S_{i} S_{j} \rightarrow V V$, which would have reduced their (co)annihilation efficiency and revived the model in the heavy mass region.

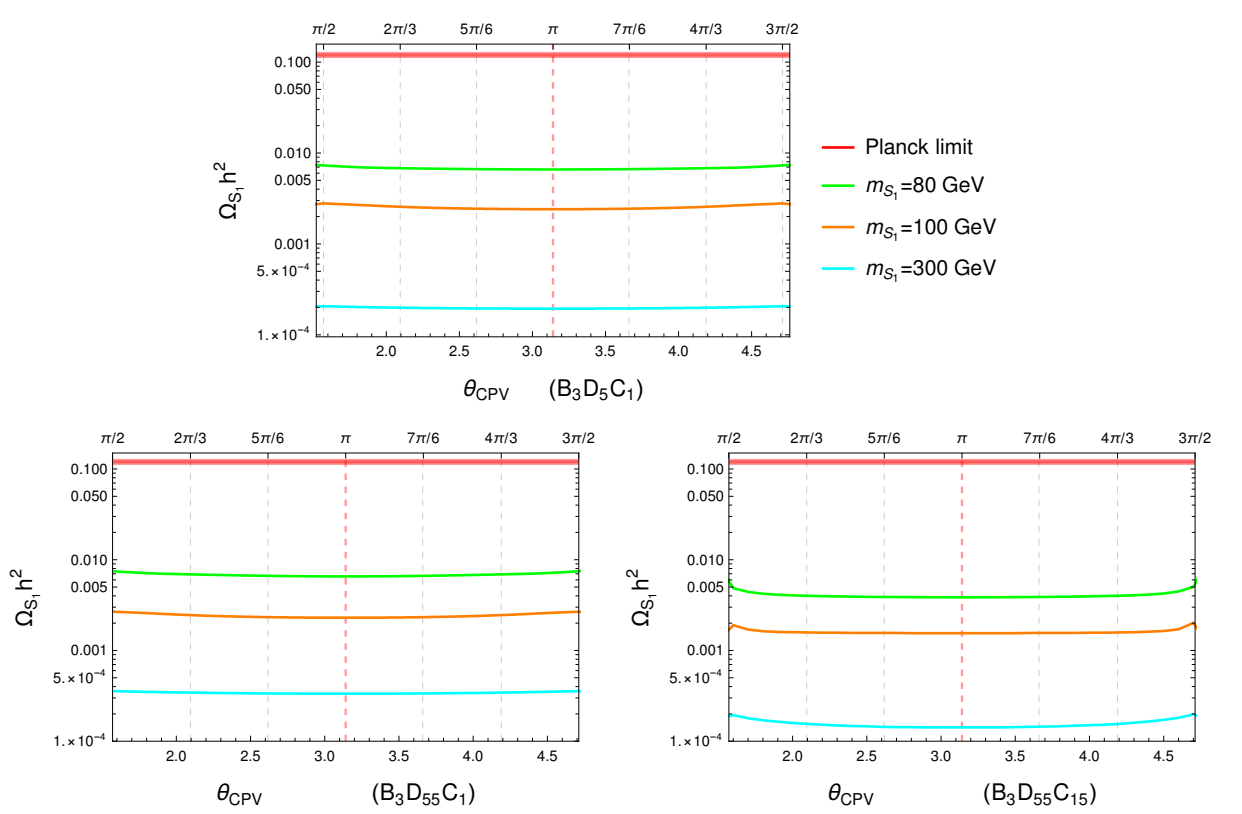

Figure 8: The change DM relic density for various DM masses with respect to the CP-violating angle. The horizontal red band shows the Planck observation limit on the abundance of DM.

\section{Conclusion and outlook}

The scalar potential is the least constrained sector of the SM which, if extended, could provide viable DM candidates, new sources of $\mathrm{CP}$ violation and a strong first order phase transition as the essential ingredients for EWBG. Of great importance are non-minimal Higgs frameworks with an extended dark/inert sector which could accommodate DM and dark CP violation unbounded by the EDM constraints, since the dark sector is protected by a conserved discrete symmetry from coupling to the SM fermions. 
We study a well-motivated 3HDM with two inert and one active doublet to play the role of the SM Higgs doublet. The dark sector interacts with the visible sector through Higgs and SM gauge bosons. The couplings through Higgs are required to be small in agreement with direct and indirect detection experiments and SM-Higgs measurements, conversely, they need to be large enough for efficient (co)annihilation of DM.

We present a novel mechanism in which the $\mathrm{CP}$ violating dark particles only interact with the SM through the gauge bosons, primarily the $Z$ boson. Such $Z$-portal dark CP violation is realised in the regions of the parameter space where Higgs-mediated (co)annihilation processes are sub-dominant and have negligible contributions to the DM relic density. We show that in these regions of the parameter space, the $Z$ portal CP violating DM can still thermalise and satisfy all experimental and observational data.

In the context of electroweak baryogenesis, the extended scalar sector could easily accommodate a strong first order phase transition. We discuss the efficient transfer of the unconstrained dark CP violation to the visible sector to source the matter-antimatter asymmetry in our upcoming publication.

\section{Acknowledgement}

The author acknowledges financial support from Academy of Finland project "Particle cosmology and gravitational waves" no. 320123 and would like to thank the organisers of the Corfu Summer Institute 2019 for the invitation and great hospitality.

\section{References}

[1] G. Aad et al. [ATLAS Collaboration], Phys. Lett. B 716, 1 (2012) [arXiv:1207.7214 [hep-ex]].

[2] S. Chatrchyan et al. [CMS Collaboration], Phys. Lett. B 716, 30 (2012) [arXiv:1207.7235 [hep-ex]].

[3] P. W. Higgs, Phys. Rev. Lett. 13, 508 (1964).

[4] F. Englert and R. Brout, Phys. Rev. Lett. 13, 321 (1964).

[5] M. Flechl [CMS Collaboration], arXiv:1905.07150 [hep-ex].

[6] G. Aad et al. [ATLAS Collaboration], Phys. Rev. D 101, no. 1, 012002 (2020) [arXiv:1909.02845 [hep-ex]].

[7] P. A. R. Ade et al. [Planck Collaboration], Astron. Astrophys. 594, A13 (2016) [arXiv:1502.01589 [astro-ph.CO]].

[8] D. E. Morrissey and M. J. Ramsey-Musolf, New J. Phys. 14, 125003 (2012) [arXiv:1206.2942 [hep-ph]].

[9] M. B. Gavela, P. Hernandez, J. Orloff and O. Pene, Mod. Phys. Lett. A 9, 795 (1994) [hep-ph/9312215].

[10] P. Huet and E. Sather, Phys. Rev. D 51, 379 (1995) [hep-ph/9404302].

[11] M. B. Gavela, P. Hernandez, J. Orloff, O. Pene and C. Quimbay, Nucl. Phys. B 430, 382 (1994) [hep-ph/9406289].

[12] K. Kajantie, M. Laine, K. Rummukainen and M. E. Shaposhnikov, Nucl. Phys. B 466, 189 (1996) [hep-lat/9510020]. 
[13] G. Jungman, M. Kamionkowski and K. Griest, Phys. Rept. 267, 195 (1996) [hep-ph/9506380].

[14] G. Bertone, D. Hooper and J. Silk, Phys. Rept. 405, 279 (2005) [hep-ph/0404175].

[15] L. Bergstrom, Rept. Prog. Phys. 63, 793 (2000) [hep-ph/0002126].

[16] O. Bertolami and R. Rosenfeld, Int. J. Mod. Phys. A 23, 4817 (2008) [arXiv:0708.1784 [hep-ph]].

[17] G. C. Branco, P. M. Ferreira, L. Lavoura, M. N. Rebelo, M. Sher and J. P. Silva, Phys. Rept. 516, 1 (2012) [arXiv:1106.0034 [hep-ph]].

[18] C. Englert, T. Plehn, D. Zerwas and P. M. Zerwas, Phys. Lett. B 703, 298 (2011) [arXiv:1106.3097 [hep-ph]].

[19] N. G. Deshpande and E. Ma, Phys. Rev. D 18, 2574 (1978).

[20] G. C. Branco, L. Lavoura and J. P. Silva, Int. Ser. Monogr. Phys. 103, 1 (1999).

[21] T. Chupp, P. Fierlinger, M. Ramsey-Musolf and J. Singh, Rev. Mod. Phys. 91, no. 1, 015001 (2019) [arXiv:1710.02504 [physics.atom-ph]].

[22] S. Inoue, M. J. Ramsey-Musolf and Y. Zhang, Phys. Rev. D 89, no. 11, 115023 (2014) [arXiv:1403.4257 [hep-ph]].

[23] V. Keus, S. F. King, S. Moretti and K. Yagyu, JHEP 1604, 048 (2016) [arXiv:1510.04028 [hep-ph]].

[24] V. Keus, N. Koivunen and K. Tuominen, JHEP 1809, 059 (2018) [arXiv:1712.09613 [hep-ph]].

[25] N. Yamanaka, B. K. Sahoo, N. Yoshinaga, T. Sato, K. Asahi and B. P. Das, Eur. Phys. J. A 53, no. 3, 54 (2017) [arXiv:1703.01570 [hep-ph]].

[26] B. Grzadkowski, O. M. Ogreid and P. Osland, Phys. Rev. D 80, 055013 (2009) [arXiv:0904.2173 [hep-ph]].

[27] P. Osland, A. Pukhov, G. M. Pruna and M. Purmohammadi, JHEP 1304, 040 (2013) [arXiv:1302.3713 [hep-ph]].

[28] A. Cordero-Cid, J. Hernandez-Sanchez, V. Keus, S. F. King, S. Moretti, D. Rojas and D. Sokolowska, JHEP 1612, 014 (2016) [arXiv:1608.01673 [hep-ph]].

[29] V. Keus, PoS CHARGED 2016, 017 (2016) [arXiv:1612.03629 [hep-ph]].

[30] A. Cordero, J. Hernandez-Sanchez, V. Keus, S. F. King, S. Moretti, D. Rojas and D. Sokolowska, JHEP 1805, 030 (2018) [arXiv:1712.09598 [hep-ph]].

[31] A. Cordero-Cid, J. Hernandez-Sanchez, V. Keus, S. Moretti, D. Rojas and D. Sokolowska, Eur. Phys. J. C 80, no. 2, 135 (2020) [arXiv:1812.00820 [hep-ph]].

[32] V. Keus, arXiv:1909.09234 [hep-ph].

[33] D. Azevedo, P. M. Ferreira, M. M. Muhlleitner, S. Patel, R. Santos and J. Wittbrodt, JHEP 1811, 091 (2018) [arXiv:1807.10322 [hep-ph]].

[34] A. Ahriche, G. Faisel, S. Y. Ho, S. Nasri and J. Tandean, Phys. Rev. D 92, no. 3, 035020 (2015) [arXiv:1501.06605 [hep-ph]].

[35] S. Choubey and A. Kumar, JHEP 1711, 080 (2017) [arXiv:1707.06587 [hep-ph]].

[36] J. O. Gong, H. M. Lee and S. K. Kang, JHEP 1204, 128 (2012) [arXiv:1202.0288 [hep-ph]].

[37] R. Howl and S. F. King, Phys. Lett. B 687, 355 (2010) [arXiv:0908.2067 [hep-ph]]. 
[38] R. Gonzalez Felipe, H. Serodio and J. P. Silva, Phys. Rev. D 87, no. 5, 055010 (2013) [arXiv:1302.0861 [hep-ph]].

[39] S. Morisi, E. Peinado, Y. Shimizu and J. W. F. Valle, Phys. Rev. D 84, 036003 (2011) [arXiv:1104.1633 [hep-ph]].

[40] G. J. Ding, S. F. King, C. Luhn and A. J. Stuart, JHEP 1305, 084 (2013) [arXiv:1303.6180 [hep-ph]].

[41] S. F. King, S. Morisi, E. Peinado and J. W. F. Valle, Phys. Lett. B 724, 68 (2013) [arXiv:1301.7065 [hep-ph]].

[42] I. P. Ivanov, V. Keus and E. Vdovin, J. Phys. A 45, 215201 (2012) [arXiv:1112.1660 [math-ph]].

[43] V. Keus, S. F. King and S. Moretti, JHEP 1401, 052 (2014) [arXiv:1310.8253 [hep-ph]].

[44] V. Keus, S. F. King, S. Moretti and D. Sokolowska, JHEP 1411, 016 (2014) [arXiv:1407.7859 [hep-ph]].

[45] V. Keus, S. F. King, S. Moretti and D. Sokolowska, JHEP 1511, 003 (2015) [arXiv:1507.08433 [hep-ph]].

[46] H. E. Haber and D. O’Neil, Phys. Rev. D 74, 015018 (2006) Erratum: [Phys. Rev. D 74, no. 5, 059905 (2006)] [hep-ph/0602242].

[47] H. E. Haber and O. Stal, Eur. Phys. J. C 75, no. 10, 491 (2015) Erratum: [Eur. Phys. J. C 76, no. 6, 312 (2016)] [arXiv:1507.04281 [hep-ph]].

[48] K. Fuyuto, X. G. He, G. Li and M. Ramsey-Musolf, arXiv:1902.10340 [hep-ph].

[49] B. Grzadkowski, O. M. Ogreid and P. Osland, JHEP 1605, 025 (2016) Erratum: [JHEP 1711, 002 (2017)] [arXiv:1603.01388 [hep-ph]].

[50] A. Cordero-Cid, J. Hernandez-Sanchez, V. Keus, S. Moretti, D. Rojas-Ciofalo and D. Sokolowska, arXiv:2002.04616 [hep-ph]. 